\title{
Stationary to nonstationary transition in crossed-field devices
}

Cite as: Phys. Plasmas 23, 033107 (2016); https://doi.org/10.1063/1.4943202

Submitted: 04 January 2016 . Accepted: 22 February 2016 . Published Online: 07 March 2016

Samuel Marini, Felipe B. Rizzato, and Renato Pakter
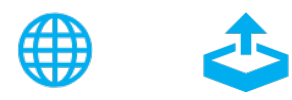

\section{ARTICLES YOU MAY BE INTERESTED IN}

Thermal effects and space-charge limited transition in crossed-field devices

Physics of Plasmas 21, 083111 (2014); https://doi.org/10.1063/1.4893313

Single electron dynamics in a Hall thruster electromagnetic field profile

Physics of Plasmas 24, 053507 (2017); https://doi.org/10.1063/1.4982685

Triplet and beam interaction in a plasma

Physics of Plasmas 24, 102124 (2017); https://doi.org/10.1063/1.5005946 


\title{
Stationary to nonstationary transition in crossed-field devices
}

\author{
Samuel Marini, Felipe B. Rizzato, and Renato Pakter \\ Instituto de Física, Universidade Federal do Rio Grande do Sul, Caixa Postal 15051, 91501-970 Porto Alegre, \\ RS, Brazil
}

(Received 4 January 2016; accepted 22 February 2016; published online 7 March 2016)

\begin{abstract}
The previous results based on numerical simulations showed that a cold electron beam injected in a crossed field gap does not reach a time independent stationary state in the space charge limited regime [P. J. Christenson and Y. Y. Lau, Phys. Plasmas 1, 3725 (1994)]. In this work, the effect of finite injection temperature in the transition from stationary to nonstationary states is investigated. A fully kinetic model for the electron flow is derived and used to determine the possible stationary states of the system. It is found that although there is always a stationary solution for any set of parameters, depending on the injection temperature the electron flow becomes very sensitive to fluctuations and the stationary state is never reached. By investigating the nonlinear dynamics of a characteristic electron, a theory based on a single free parameter is constructed to predict when the transition between stationary and nonstationary states occurs. In agreement with the previous numerical results, the theory indicates that for vanishing temperatures the system never reaches the time independent stationary state in the space charge limited regime. Nevertheless, as the injection temperature is raised it is found a broad range of system parameters for which the stationary state is indeed attained. By properly adjusting the free parameter in the theory, one can be able to describe, to a very good accuracy, when the transition occurs. (C) 2016 AIP Publishing LLC.

[http://dx.doi.org/10.1063/1.4943202]
\end{abstract}

\section{INTRODUCTION}

The understanding of the characteristics of the electron flow in the presence of crossed electric and magnetic fields is fundamental for the development of many advanced applications in different areas. For such devices working at low currents, it is shown that if a sufficiently strong magnetic field is imposed, the electrons emitted from the cathode will not reach the anode. ${ }^{1}$ Instead, they will perform a cycloidal motion that will eventually lead them back to the cathode. ${ }^{2,3}$ As the current is increased and the self-fields due to the electrons become more intense, an interesting new phenomenon may occur. For instance, in the presence of fluctuations the cycloidal trajectories may become unstable if there are particles that are injected with vanishing small velocities. What happens is that these particles may loose energy while transiting in the cathode-anode gap and fail to return to the cathode at the end of their cycloidal trajectory. ${ }^{4-6}$ They then become trapped in the gap region leading to an increasing charge build up in the system.

At high currents, the self-fields may also be responsible for the onset of a space-charge limited regime, where the accelerating field is completely shielded at the cathode by the electron distribution present in the gap region. ${ }^{7,8}$ This corresponds to the formation of a virtual cathode ${ }^{9}$ that prevents new particles from entering the device. Numerical simulations considering cold (monoenergetic) injected beams have shown that the space-charge limit imposes a drastic change in the electron flow: while it is stationary below the limiting current, it becomes turbulent (nonstationary) above it. ${ }^{3}$ More recently, however, it has been found that thermal effects may play a major role in the electron dynamics in a crossed field gap. ${ }^{6}$ In particular, it has been detected the occurrence of stationary solutions for currents well beyond the space-charge limiting threshold.

Our aim here is to investigate in detail the onset of nonstationary regimes when thermal effects are taken into account. We consider a fully kinetic Vlasov-Poisson description for the electron flow and search for its stationary solutions. Different from the cold case where the electron dynamics is dictated by a linear equation of motion, ${ }^{2,3}$ in the thermal case the characteristic trajectory is given by a nonlinear equation. To theoretically investigate its properties, we then derive an effective potential ${ }^{10}$ for the evolution of the characteristic momentum. We find that for sufficiently low temperatures the effective potential forms a well that nearly confines the characteristic trajectory. In such cases, it is anticipated that the trajectories will be very sensitive to any small fluctuation in the potential which may lead to trapping and untrapping of particles, preventing the system to reach the predicted stationary solution. This scenario is confirmed by self-consistent numerical simulations which show the existence of a threshold temperature below which the stationary solution is never reached.

\section{MODEL}

We consider a planar gap whose cathode (anode) is oriented along the $x-z$ plane and is located at $y=0(y=L)$. The gap is immersed in a uniform transverse magnetic field $\mathbf{B}_{0}=-B_{0} \hat{\mathbf{z}}$ and subject to a potential difference $V_{0}$ between the electrodes that generates a uniform electric field $\mathbf{E}_{0}=-\left(V_{0} / L\right) \hat{\mathbf{y}}$. The electrons are accelerated along the $y$ direction by $\mathbf{E}_{0}$ and deflected clockwise by $\mathbf{B}_{0}$. When $B_{0}$ is large enough, the electron will not gain sufficient energy to reach the anode and will be pushed back to the cathode by 
the magnetic force, performing the typical cycloidal trajectory shown in Fig. 1. If $B_{0}$ prevents all the particles from reaching the anode and there is no net current between the electrodes, we say, that the gap is magnetic insulated. We focus our analysis on this particular regime.

In the absence of charge in the gap, the electric potential is simply given by that of a planar capacitor in free space, namely, $\phi(y)=E_{0} y$. However, as charge builds up in the system, the self-fields will become important and the electric potential will be a function of the particle density $n(y)$ as given by the Poisson's equation,

$$
\frac{\partial^{2} \phi}{\partial y^{2}}=\frac{e}{\epsilon_{0}} n(y)
$$

where $\epsilon_{0}$ is the vacuum permittivity, $-e$ is the electron charge, and the potential satisfies the boundary conditions $\phi(0)=0$ and $\phi(L)=V_{0}$. The dynamics of a given electron subject to such crossed-fields is dictated by the Hamiltonian ${ }^{6}$

$$
H=\frac{1}{2 m}\left[p_{y}^{2}+e^{2} B_{0}^{2} y^{2}\right]-e \phi(y)
$$

where $m$ is the electron mass and, for simplicity, we have assumed that the electrons are injected with velocities normal to the cathode, such that the conserved canonical momenta along $x$ and $z$ satisfy $p_{x}=p_{z}=0$. We have also assumed that the gap region is sufficiently thin such that we can safely neglect the self-magnetic fields. ${ }^{11}$ The electron dynamics is given by the Hamilton equations $\dot{y}=\partial H / \partial p_{y}$ and $\dot{p}_{y}=-\partial H / \partial y$, where the dot stands for time derivative. From the single particle Hamiltonian in Eq. (2), we can then describe the evolution of the whole electron distribution as described by the distribution function in phase space $f\left(y, p_{y}, t\right)$, which evolves according to the Vlasov equation ${ }^{9}$

$$
\frac{d f}{d t}=\frac{\partial f}{\partial t}+\frac{\partial H}{\partial p_{y}} \frac{\partial f}{\partial y}-\frac{\partial H}{\partial y} \frac{\partial f}{\partial p_{y}}=0 .
$$

An important consequence of the Vlasov equation is that it shows that the system evolves over the phase space as an incompressible fluid because the convective derivative of $f\left(y, p_{y}, t\right)$ vanishes. This feature will be directly employed in order to determine the stationary states of the system. The particle density is related to the distribution function in phase space by $n=\int f\left(y, p_{y}, t\right) d p_{y}$. Substituting this expression in Eq. (1), we obtain the Vlasov-Poisson closed set of equations that fully describe the electron flow in the gap region.

\section{STATIONARY SOLUTIONS}

The analysis performed so far on the onset of turbulence in crossed-field gaps considered only the cases where the

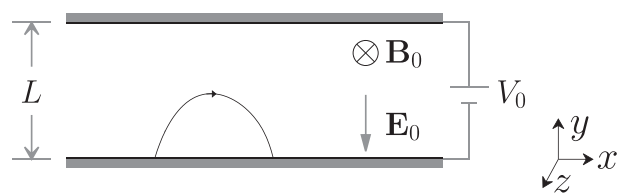

FIG. 1. A schematic diagram of a planar crossed-field gap. A typical cycloidal trajectory of an electron is also shown. electron injection was cold,i.e., all the electrons entered in the gap region with the same velocity. ${ }^{3-5}$ In order to investigate the role of injection temperature in the onset of turbulence, we will consider that the electrons have a velocity distribution with a finite spread at the cathode. More specifically, we will assume that they are injected at $y=0$ as a waterbag in momentum: $p_{y}$ is uniformly distributed from 0 to $p_{0}$. This corresponds to impose that the distribution function satisfies a boundary condition of the form $f\left(y=0, p_{y}\right)$ $=n_{0} / p_{0}$ for $0 \leq p_{y} \leq p_{0}$, where $n_{0}$ is the particle density at the cathode. In fact, the technique employed here to determine stationary solutions for the electron flow can be generalized to other velocity distributions by approximating them by a series of waterbags. ${ }^{12}$ Nevertheless, it is anticipated that a single waterbag already captures the relevant features of the emission of a hot cathode.

Once a stationary solution is attained, quantities like the distribution function, the particle density, and the electrostatic potential become time independent. Hence, the single particle Hamiltonian (2) also becomes a constant of motion, corresponding to the total energy of the particle. Because $\phi(0)=0$, the energy for the most energetic electron-the one that is injected from the cathode with momentum $p_{0}$-is $H_{\max }=p_{0}^{2} / 2 m$. From energy conservation, we can readily write its momentum as a function of the position as

$$
p_{\max }(y)= \pm\left[p_{0}^{2}+2 e m \phi(y)-e^{2} B_{0}^{2} y^{2}\right]^{1 / 2},
$$

where the plus (minus) sign refers to the electron moving towards the anode (cathode). Figure 2 illustrates this curve in the phase-space. Since at the anode $\phi(L)=V_{0}$, it is clear from Eq. (4) that whenever

$$
B_{0}>\frac{\left(p_{0}^{2}+2 e m V_{0}\right)^{1 / 2}}{e L},
$$

not even the most energetic electron will reach the anode. Instead, it will perform a cycloidal motion like the one depicted in Fig. 1. Therefore, Eq. (5) corresponds to the magnetic insulation regime condition where all the electrons return to the cathode.

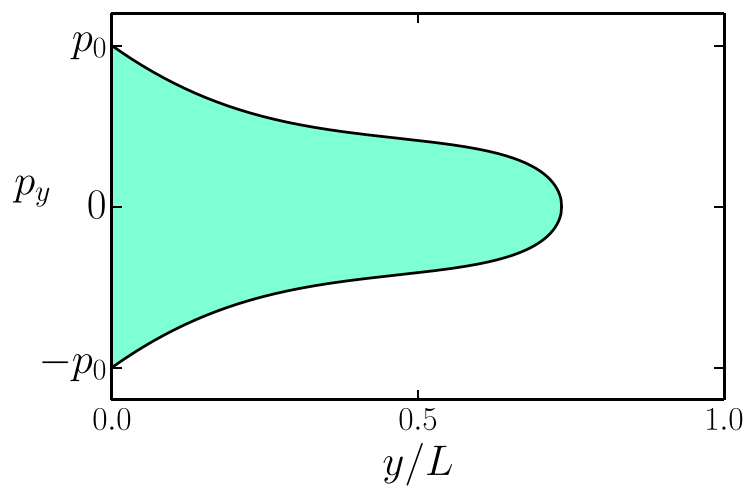

FIG. 2. Phase space portray showing $p_{\max }(y)$ (thick solid line), Eq. (4), for a magnetic insulated case. It corresponds to the trajectory of the most energetic particle, whose energy is $H\left(y, p_{y}\right)=H_{\max }$. The stationary distribution of Eq. (6) is uniformly distributed with density $n_{0} / p_{0}$ inside this curve (colored area). 
In order to determine the stationary state, we need to understand how the charge buildup occurs in the system. As the particles are injected in an empty gap, they start performing their respective cycloidal motion. Because the system is still far from being stationary the electrostatic potential varies with time such that particles may loose or gain energy as they move in the gap. This particularly affects the low energy particles-those injected with $p_{y} \approx 0$ - because if they loose energy they may fail to return to the cathode, becoming trapped in the gap region. ${ }^{4-6}$ This causes a continuous increase in the charge accumulated inside the gap. At this point, the incompressibility in the evolution of the Vlasov equation becomes crucial because it sets a limit on the maximum density of particles found in the phase space which has to be always equal or smaller (in a coarse grained sense $^{13}$ ) than the density at the injection, namely, $n_{0} / p_{0}$. Note that the incompressibility condition plays no role when one considers monoenergetic cold beams because their injection density in phase space is divergent; however, it does play a major role when one considers a more realistic thermal injection. Hence, the charge keeps increasing in the system up to the point where the available phase space is completely filled by the particles. ${ }^{6}$ The available phase space corresponds to the region where the energy does not exceed $H_{\text {max }}$. The stationary distribution function is then given by

$$
f_{s}\left(y, p_{y}\right)=\frac{n_{0}}{p_{0}} \Theta\left[H_{\max }-H_{\left.\left(y, p_{y}\right)\right]}\right.
$$

where $\Theta$ is the Heaviside function (see Fig. 2). Note that $f_{s}\left(y, p_{y}\right)$ is effectively a stationary solution of the Vlasov equation because it only depends on the phase space variables through the Hamiltonian $H\left(y, p_{y}\right)$ which is a conserved quantity for the single particle dynamics after the stationary state is reached. Integrating $f_{s}$ over the momentum space, we obtain the stationary particle density as given by

$$
n(y)=2 \frac{n_{0}}{p_{0}}\left|p_{\max }(y)\right|,
$$

where the factor " 2 " accounts for the particles that are moving both to and from the cathode. Substituting Eqs. (4) and (7) in the Poisson's equation (1), we then obtain a closed equation for stationary self-field electric potential, which has to be numerically solved. In practice, we solve the Poisson's equation as an initial value problem by setting $\phi(0)=0$ and taking a guess value for the electric field at the cathode $E_{c}=-\partial \phi /\left.\partial y\right|_{y=0}$. Using an iterative method, we find a value for $E_{c}$ that satisfies the correct boundary condition at the cathode $\phi(L)=V_{0}$.

It is convenient to define the dimensionless parameters $\nu_{0}=2 m V_{0} / e B_{0}^{2} L^{2}, \eta_{0}=e n_{0} L^{2} / \epsilon_{0} V_{0}$, and $T_{0}=p_{0}^{2} / 12 e^{2} B_{0}^{2} L^{2}$ that correspond, respectively, to a scaled ratio between electric and magnetic force in the gap, a normalized electron density, and a normalized temperature (momentum spread) at injection. In terms of these parameters, the magnetic insulation condition (5) becomes $\nu_{0}+12 T_{0}<1$. In the remainder of the paper, we will consider $\nu=0.8$, setting as the upper limit for the temperature $T_{0}<0.016$. In Fig. 3, we plot the cathode electric field for the stationary state as a

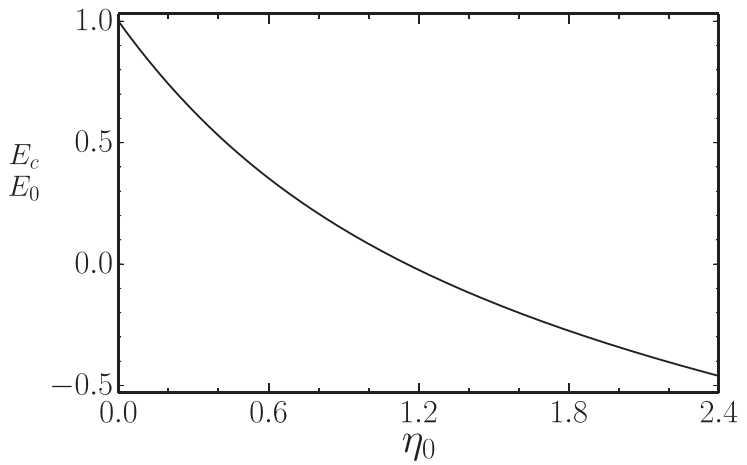

FIG. 3. Cathode electric field of the stationary state obtained from the theory as a function of the normalized electron density. The remainder parameters are $\nu_{0}=0.8$ and $T_{0}=0.0075$. The electric field is normalized to the vacuum one $E_{0}=-V_{0} / L$.

function of the normalized electron density for $T_{0}=0.0075$. As expected, for low charge densities $\left(\eta_{0} \approx 0\right)$ the cathode field is very little affected by the space charge and it is approximately equal to the vacuum accelerating field $E_{0}$. As $\eta_{0}$ increases, $E_{c} / E_{0}$ falls because the space charge effects become progressively more important, partially shielding the accelerating field. At $\eta_{0} \approx 1.2$, the space charge limiting case with $E_{c}=0$ is reached. Beyond this point, the self-field generated by the charge distribution in the gap becomes larger than the accelerating field as indicated by $E_{c} / E_{0}<0$ and particles are pushed back to the cathode as soon as they are injected in the system.

We have considered other values of the injection temperature $T_{0}$ and the trend that we find is the same as the one shown in Fig. 3. Namely, there is always one stationary solution for a given set of parameters ${ }^{14}$ and it goes from accelerating with $E_{c} / E_{0}>0$ to space charge limited with $E_{c} / E_{0}<0$ as the particle density $\eta_{0}$ is increased. In Fig. 4, the thick solid curve shows where the transition between these regimes occurs in the $\eta_{0}$ vs. $T_{0}$ parameter space. In particular, the space charge limited region to the right of the curve is where the turbulent states are found in the cold injection case. The fact that we can always find stationary solutions in this parameter region for the thermal case does not a priori guarantee that the system will not present a

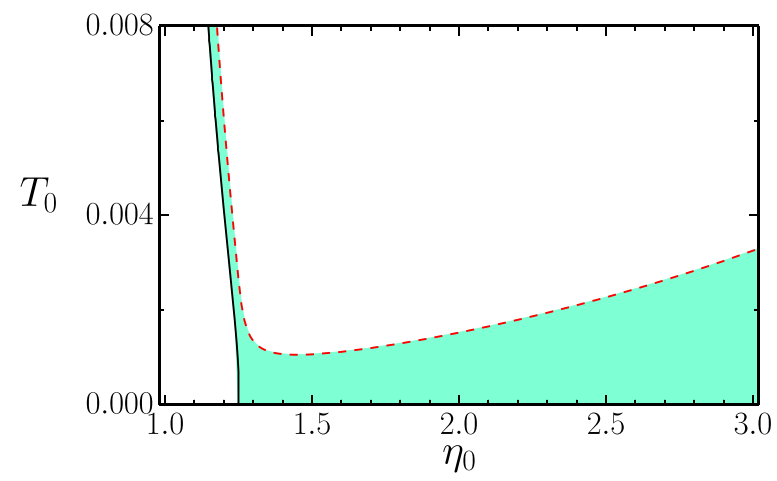

FIG. 4. Parameter space $\eta_{0}$ vs. $T_{0}$. The solid curve corresponds to the points where the cathode electric field vanishes, $E_{c}=0$, and marks the transition between accelerating (smaller $\eta_{0}$ ) and space charge limited (larger $\eta_{0}$ ) regimes. The dashed curve shows the points where $\Delta\left(\eta_{0}, T_{0}\right)=\Delta_{c}$, with $\Delta_{c}=0.02$. The colored region represents the points where $0 \leq \Delta \leq \Delta_{c}$. 
turbulent (nonstationary) character. In fact, the stationary solution may be unstable ${ }^{15}$ or very sensitive to small oscillations in the system in such a way that it may not be actually attained in practice.

\section{ELECTRON DYNAMICS AND EFFECTIVE POTENTIAL}

It is not an easy task to perform a fully kinetic linear stability analysis of the stationary solutions. Moreover, the linear stability may not even be determinant to specify the occurrence of turbulence because the system starts very far from the stationary solution and may never reach its close vicinity to be affected by its linear stability. Instead, we will perform a detailed nonlinear analysis of the electron dynamics in the gap in order to determine when it is more or less robust against fluctuations in the system. We focus on the space charge limited parameter region where the turbulent solutions were found.

Let us consider the dynamics of the most energetic particle. Taking two derivatives of Eq. (4) with respect to time, we can obtain a closed equation for the evolution of $p_{\max }$ which reads

$$
\ddot{p}_{\max }+\left(\Omega_{e}^{2}-\frac{2 \omega_{e}^{2}}{p_{0}}\left|p_{\max }\right|\right) p_{\max }=0,
$$

where $\Omega_{e}=e B_{0} / m$ and $\omega_{e}=\left(e^{2} n_{0} / \epsilon_{0} m\right)^{1 / 2}$ are electron cyclotron and plasma frequencies, and applied Eqs. (1) and (7) and the fact that $d y / d t=p_{\max } / m$ along the particle trajectory. Equation (8) is equivalent to Llewellyn form ${ }^{2,16}$ for a thermal crossed field gap. Different from the cold case, this equation is now nonlinear and, in principle, can only be solved numerically. However, even without finding its explicit solution, we can already discover important properties of the particle trajectory by constructing an effective potential for its momentum evolution. ${ }^{10}$ Conveniently multiplying Eq. (8) by $2 \dot{p}_{\max } / \Omega_{e}^{2} p_{0}^{2}$, we can readily integrate it to obtain

$$
\left(\frac{\dot{p}_{\max }}{\Omega_{e} p_{0}}\right)^{2}+\mathcal{V}\left(p_{\max }\right)=\mathcal{E} .
$$

In the equation above, $\mathcal{V}\left(p_{\max }\right)$ is an effective potential driving $p_{\max }$ dynamics, which can be written as

$$
\mathcal{V}\left(p_{\max }\right)=\left(1-\frac{2 \nu_{0} \eta_{0}}{3}\left|\frac{p_{\max }}{p_{0}}\right|\right)\left(\frac{p_{\max }}{p_{0}}\right)^{2},
$$

and $\mathcal{E}$ is an integration constant that plays the role of an effective energy for the trajectory. Evaluating Eq. (9) at the injection where $p_{\max }=p_{0}$ and $\dot{p}_{\max }=-e E_{c}$, we can compute the value of the effective energy as

$$
\mathcal{E}=\frac{\nu_{0}^{2}}{48 T_{0}}\left(\frac{E_{c}}{E_{0}}\right)^{2}+1-\frac{2 \nu_{0} \eta_{0}}{3} .
$$

The shape of the effective potential is shown by the thick solid curve in Fig. 5 for the choice of parameters $\nu_{0} \eta_{0}$ $=1.5$ (a) and $\nu_{0} \eta_{0}=0.95$ (b). Both cases present a local
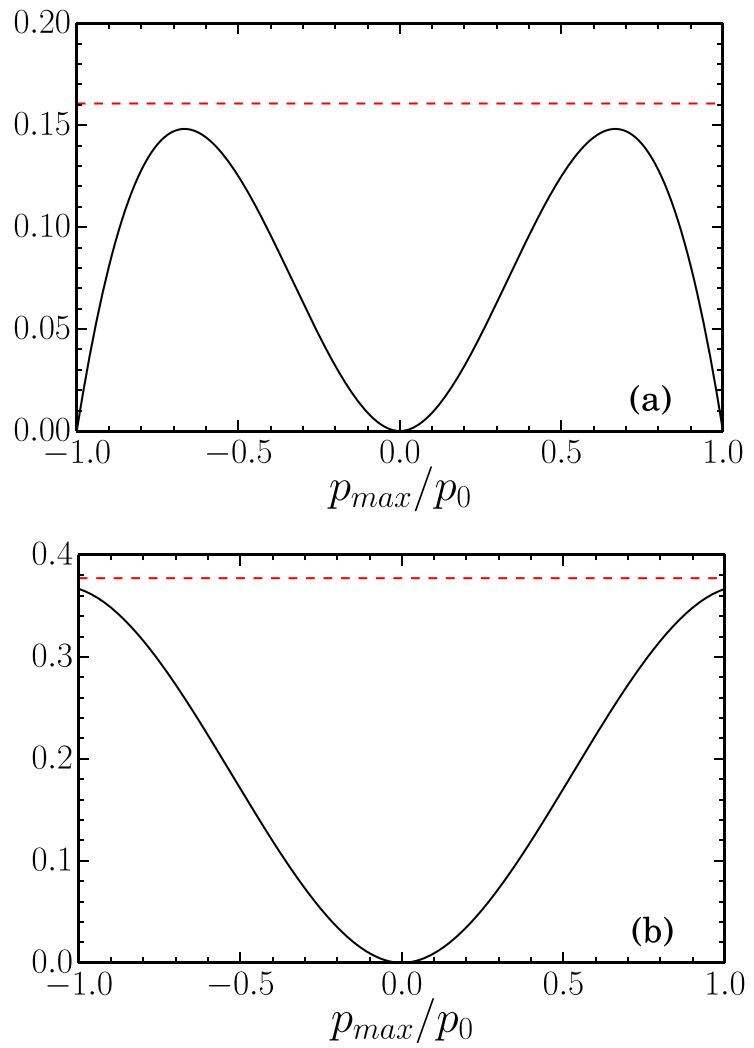

FIG. 5. Plots of the effective potential (solid curve) for $\nu_{0} \eta_{0}=1.5$ (a) and $\nu_{0} \eta_{0}=0.95$ (b). The dashed lines show the corresponding values of the effective energy for $T_{0}=0.0075$.

minimum at $p_{\max }=0$. For the parameters of panel (a), there are also two symmetric maximums. With the aid of Eq. (10), we find that the maximums are located at $p_{\max }= \pm \bar{p}_{\max }$ with $\bar{p}_{\max } \equiv p_{0} / \nu_{0} \eta_{0}$. The maximums are absent from panel (b) because for this case $p_{0}<\bar{p}_{\max }$. It is also shown in the figure the straight line that represents the value of the effective energy for $T_{0}=0.0075$. For the trajectories shown, the particle is injected with $p_{\max }=+p_{0}$ and starts decreasing its momentum-moving to the left in the figure-because $\dot{p}_{\max }<0$ at the cathode (space charge limited case). Since $\mathcal{E}$ $>\mathcal{V}\left(p_{\max }\right)$ for all $p_{\max }$, there are no turning points where $\dot{p}_{\max }$ changes its sign [see Eq. (9)]. Hence, $p_{\max }$ continuously decreases until the particle hits the cathode again with a momentum $p_{\max }=-p_{0}$ and leaves the system.

A quick inspection on Eq. (11) reveals that the only explicit dependence of $\mathcal{E}$ on $T_{0}$ appears in the first term on the rhs and is such that the effective energy would decrease with an increase in the temperature. However, one should notice that the cathode electric field is also a function of the injection temperature. In fact, by solving the stationary state equations derived in Sec. III one finds that $E_{c}$ tends to increase with $T_{0}$ and that its variation dominates over the $1 / T_{0}$ term of Eq. (11). Hence, by decreasing the cathode temperature the effective energy also decreases and the straight lines of Fig. 5 moves downward, closer to the effective potential curves. Nevertheless, except for the transition line where $E_{c}=0$ (see Fig. 4$), \mathcal{E}$ is always above $\mathcal{V}\left(p_{\max }\right)$ and there are no turning points for a strictly stationary solution. 
In practice, however, as the system relaxes it presents fluctuations that will cause both $\mathcal{E}$ and $\mathcal{V}\left(p_{\text {max }}\right)$ to vary in time. If at a certain instant $\mathcal{E}=\mathcal{V}\left(p_{\text {max }}\right)$, there will be a change in the sign of $\dot{p}_{\max }$ and the particle momentum will start bouncing inside the effective potential well, which may prevent the system from reaching the time independent stationary state. It is expected that the smaller is the difference between the theoretically predicted values of $\mathcal{E}$ and $\mathcal{V}\left(p_{\text {max }}\right)$, the more likely is such an event. We thus define

$$
\Delta=\min \left[\mathcal{E}-\mathcal{V}\left(p_{\max }\right)\right]
$$

where the minimization is to be taken along the stationary solution trajectory $-p_{0} \leq p_{\max } \leq p_{0}$. The smallness of $\Delta$ is foreseen to serve as an indicator of how sensitive the stationary solution is to the fluctuations. It is clear from Fig. 5(a) that when $\nu_{0} \eta_{0}>1$ and the maximums of the effective potential are present that $\Delta=\mathcal{E}-\mathcal{V}\left(\bar{p}_{\text {max }}\right)$. On the other hand, when $\nu_{0} \eta_{0}<1$, such as in Fig. 5(b), the minimization in Eq. (12) leads to $\Delta=\mathcal{E}-\mathcal{V}\left(p_{0}\right)$. Using these expressions, we can determine $\Delta$ for any given set of parameters.

To perform a qualitative analysis of the regions in the parameter space where the system is more sensitive to fluctuations, we choose a small number $\Delta_{c}$ and determine from the expressions above when $\Delta=\Delta_{c}$. As an illustration, the dashed curve in Fig. 4 shows the result for $\Delta_{c}=0.02$. The colored area in the figure thus represents when $\Delta$ is sufficiently small to satisfy $0<\Delta<\Delta_{c}$ and corresponds to a region where the onset of turbulence is more likely. For high injection temperatures (above $T_{0} \sim 0.003$ ), we notice that this region is limited to a very thin layer near the transition to the space charge limited regime. This means that for these temperatures the system is expected to reach the stationary solution for most of the injection current densities $\eta_{0}$ and turbulence is not foreseen. On the other hand, for low temperatures we see that $0<\Delta<\Delta_{c}$ is satisfied for an extended region of the parameter space where turbulence is expected. In particular, we see that as $T_{0} \rightarrow 0$ the system is not expected to reach a time independent stationary state for any space charge limited case, which is in agreement with the simulation results. ${ }^{3}$ By considering other small values of $\Delta_{c}$ leads to the same qualitative profile for the $\Delta=\Delta_{c}$ curve. The actual value of $\Delta_{c}$ will be resolved in Section $\mathrm{V}$.

\section{SIMULATION RESULTS}

To verify the theoretical predictions above, we run $N$ particle self-consistent simulations. To model the charging process in real devices, in the simulations we start with an empty gap and continuously inject particles from the cathode with transverse momenta uniformly distributed from 0 to $p_{0}$. The dynamics of a given particle inside the gap region is derived from the Hamiltonian (2) where the self-fields are calculated using Green's functions. ${ }^{17}$ In Fig. 6, we plot the evolution of the force acting upon a test particle fixed at $y / L=0.35$ as obtained from the simulations with $\eta_{0}=3.0$ (space charge limited regime) and 2 different $T_{0}$. We see that for both injection temperatures the force undergoes violent oscillations in the beginning. However, for the higher $T_{0}$ case (dashed curve) it eventually saturates to a nearly

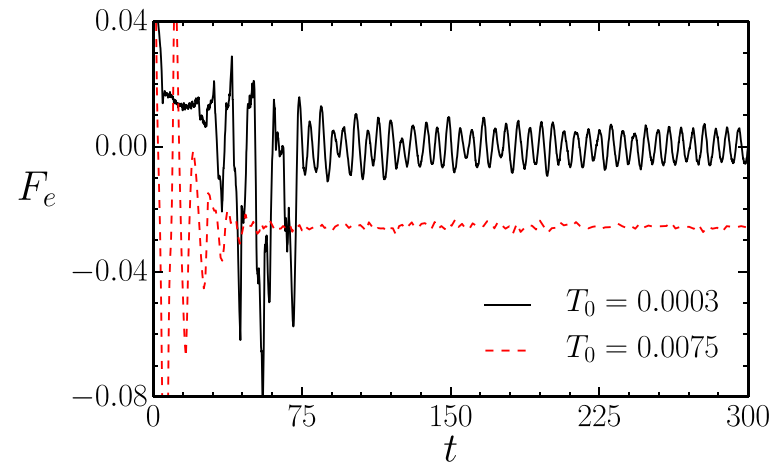

FIG. 6. Time evolution of the net force acting upon a test particle fixed at $y / L=0.35$ as obtained from self-consistent simulations in the space charge limited regime. For $T_{0}=0.0075$ (dashed curve), the force eventually reaches a stationary value that agrees with the theoretically predicted one, whereas for $T_{0}=0.0003$ (solid curve) the force shows persistent oscillations. The remainder parameters are $\eta_{0}=3.0$ and $\nu_{0}=0.8$.

constant value indicating that the system has reached the stationary state. In fact, the stationary value of the force at $y / L=0.35$ calculated from the theory is $F_{e}=-0.0255$, which is in excellent agreement with the one obtained in the simulation. Note that the force is negative because in a space charge limited stationary state all the particles are pushed back towards the cathode. We have evaluated $F_{e}$ at different positions and they all relax to constant values that match with the theoretically predicted ones. On the other hand, for the lower temperature (solid curve) the solution presents persistent oscillations around $F_{e} \approx 0$ that do not fade and prevent the system from attaining the stationary state. These oscillations are clearly related to the changes in the sign of $\dot{p}_{\text {max }}$ as discussed in the previous section.

Based on the results of Fig. 6, we notice that the turbulent state is characterized by the occurrence of a positive force acting upon the beam particles after the initial transient time. Hence, in order to determine when the stationarynonstationary transition occurs, we ran simulations for times longer than the transient time with a given $\eta_{0}$ and increased $T_{0}$ from 0 at small increments until the condition $F_{e}<0$ was found for all the particles, indicating that a time-independent stationary regime has emerged. The results obtained for

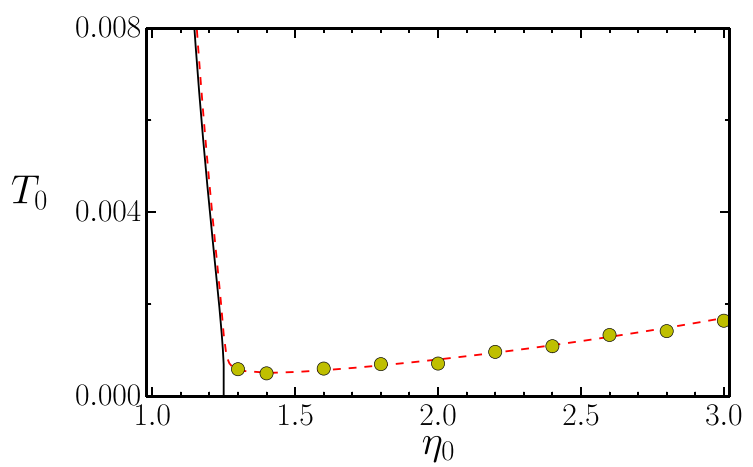

FIG. 7. Parameter space $\eta_{0}$ vs. $T_{0}$. The symbols show the injection temperature above which the system attains a time-independent stationary state in the self-consistent simulations for the given $\eta_{0}$. The dashed curve corresponds to the theoretical curve $\Delta\left(\eta_{0}, T_{0}\right)=\Delta_{c}$ obtained with $\Delta_{c}=0.006$ The solid curve marks the transition between accelerating and space charge limited regimes. 
different values of $\eta_{0}$ are shown in Fig. 7, where the symbols represent the injection temperature above which the system attains the time-independent stationary state. To test the theory presented in Sec. IV, we search for a $\Delta_{c}$ that best fits the numerical results. We find $\Delta_{c}=0.006$ which is shown by the dashed curve in Fig. 7. The very good agreement is a clear indication that the mechanism discussed in Sec. IV is the one responsible for the onset of turbulence at low temperatures. As a reference, it is also shown in the figure the curve that marks the transition to the space charge limited regime (solid curve). For larger temperatures, we notice that the dashed and the solid curves almost coincide, meaning that the region where the theory predicts the onset of nonstationary states is effectively very narrow. As a matter of fact, we were unable to identify nonstationary solutions in this region in the numerical simulations.

\section{CONCLUSIONS}

We have investigated temperature effects in the transition between stationary and nonstationary states in the relaxed state of the electron flow in crossed field gaps. A fully kinetic Vlasov-Poisson model was derived and used to determine the stationary states of the system. It is found that the theory predicts the existence of a stationary solution for any set of system parameters. However, in the space-charge limited regime and depending on the injection temperature, the electron flow becomes very sensitive to fluctuations such that the stationary state is never reached and the system was found to present persistent oscillations. By investigating the nonlinear dynamics of the most energetic electron, we were able to estimate when the system is more sensitive to the fluctuations and to determine the mechanism that prevents it from reaching the stationary state. In particular, we constructed a theory based on a single free parameter to predict the occurrence of the transition between stationary and nonstationary states. It is found that for vanishing temperatures the system never reaches the time independent stationary state in the space charge limited regime, which is in agreement with the previous results. ${ }^{3}$ Nevertheless, as the temperature is raised, we find a broad range of system parameters for which the stationary state is indeed attained and is in agreement with the one predicted by the theory. Finally, by properly adjusting the free parameter in the theory we were able to describe to a very good accuracy the transition curve between the stationary and the nonstationary states.

\section{ACKNOWLEDGMENTS}

This work was partially supported by $\mathrm{CNPq}$ and FAPERGS, Brazil, and by the US-AFOSR under the Grant No. FA9550-12-1-0438.

${ }^{1}$ A. W. Hull, Phys. Rev. 18, 31 (1921)

${ }^{2}$ Y. Y. Lau, P. J. Christenson, and D. Chernin, Phys. Fluids B 5, 4486 (1993).

${ }^{3}$ P. J. Christenson and Y. Y. Lau, Phys. Plasmas 1, 3725 (1994).

${ }^{4}$ P. J. Christenson and Y. Y. Lau, Phys. Rev. Lett. 76, 3324 (1996).

${ }^{5}$ P. J. Christenson, D. P. Chernin, A. L. Garner, and Y. Y. Lau, Phys. Plasmas 3, 4455 (1996).

${ }^{6}$ S. Marini, F. B. Rizzato, and R. Pakter, Phys. Plasmas 21, 083111 (2014).

${ }^{7}$ C. D. Child, Phys. Rev. 32, 492 (1911); I. Langmuir, ibid. 21, 419 (1923).

${ }^{8}$ K. G. Kostov and J. J. Barroso, Phys. Plasmas 9, 1039 (2002).

${ }^{9}$ R. C. Davidson, Physics of Nonneutral Plasmas (Imperial College Press, London, 2001)

${ }^{10}$ R. Pakter, R. S. Schneider, and F. B. Rizzato, Phys. Rev. E 49, 1594 (1994).

${ }^{11}$ G. H. Goedecke, B. T. Davis, C. Chen, and C. V. Baker, Phys. Plasmas 12, 113104 (2005)

${ }^{12}$ Y. Levin, R. Pakter, and T. N. Teles, Phys. Rev. Lett. 100, 040604 (2008); T. N. Teles, R. Pakter, and Y. Levin, Appl. Phys. Lett. 95, 173501 (2009).

${ }^{13}$ A. Campa, T. Dauxois, and S. Ruffo, Phys. Rep. 480, 57 (2009); Y. Levin, R. Pakter, F. B. Rizzato, T. N. Teles, and F. P. da C. Benetti, Phys. Rep. 535, 1 (2014).

${ }^{14}$ In Ref. 6, it is reported the occurrence of multiple solutions for some parameters. But they only occur if the beam is injected with momentum distribution that satisfies $p_{0 \min } \leq p_{y} \leq p_{0 \max }$ with nonvanishing value of

$p_{0 \min }$.
${ }^{15}$ M. V. Alves, G. Gnavi, F. T. Gratton, S. Ferro, and C. H. Moreno, Phys. Plasmas 7, 2798 (2000).

${ }^{16}$ C. K. Birdsall and W. B. Bridges, Electron Dynamics of Diode Regions (Academic, New York, 1966).

${ }^{17}$ F. B. Rizzato, R. Pakter, and Y. Levin, Phys. Rev. E 80, 021109 (2009). 